\title{
Virtual reality-helping fight psychological barriers and dependence post stroke
}

\begin{abstract}
Objective: To investigate if virtual reality will improve balance and reduce fear of fall in stroke subject.

Design: Single case, A-B-A design.

Setting: Riphah Rehab and Research Centre, Pakistan Railway Hospital

Participant: A 68 year old man having left sided hemiplegia that effected balance and ambulation.

Intervention: The subject was trained using Xbox 360. Training was given for 8 weeks. 3 rounds of 20,000 leaks game each having 3 waves with resting period of $5 \mathrm{~min}$, was played by the patient.

Main Outcome Measures: Berg Balance Scale, Time up and Go Test, Five times sit to stand test and fall efficacy Scale.

Results: Results showed obvious improvement in score of berg balance scale and fall efficacy scale. Five times sit to stand and time up and go scores also showed to be improved. Subject was ambulating independently after 8 weeks of intervention with full confidence and traveling in local transport alone. Patient also reported that he performs day to day tasks without any fear of fall.
\end{abstract}

Conclusion: Virtual training improves balance and helps to reduce fear of fall promoting independent lifestyle.

Keywords: Balance, Stroke, Virtual reality, Fear of fall
Volume 7 Issue 2 - 2017

Qurat-ul- ain

Demonstrator, Riphah International University, Pakistan

Correspondence: Qurat-ul-ain, Demonstrator, Riphah International University, Riphah Rehab and Research Centre Pakistan Railway Hospital, Pakistan, Tel 0336-5540062 Email deem1970@gmail.com

Received: November 20, 2016 | Published: January 27, 2017

\section{Abbreviations} Reality

FES: Fall Efficacy Scale, BBS: Berg balance scale, VR: Virtual

\section{Introduction}

Cerebro-vascular accident is a common neurological insult that ends up in a variety of signs and symptoms including hemi paresis, motor impairment, balance deficits, speech disorders, dyspraxia, cognitive impairments, dysesthesia, and visual problems. Motor and balance impairments are prime obstacle in performing activities of daily living thus making stroke leading cause of disability. Tyson SF et al. in their study found that out of total stoke survivors worldwide $25-74 \%$ is completely and partially dependent on assistance to perform their routine life activities after stroke. ${ }^{1}$ It is need of the hour to focus on balance and gait training in stroke survivors to bring them back towards independent life. Different approaches and techniques are being used for balance training. Training program can be more advantageous if it is appealing and motivating for the patient. ${ }^{2}$ Currently Virtual reality has emerged as an effective treatment approach in stroke rehabilitation as it is designed to be more interesting in comparison with traditional programs. ${ }^{2}$ It is a computer generated interface that implements real-time simulation of an activity or environment allowing user interaction. ${ }^{3}$

\section{Methods}

\section{Case description}

The patient was a 68 year old man. He had stroke attack 1 day back. He had left hemiplegia due to infarct in right MCA, diagnosis was confirmed by computed tomography scan. The patient was in flaccid state later followed by hemiparesis. After two weeks gradually muscle tone improved and patient was able to perform certain activities and able to ambulate with assistance. According to berg balance scale the score was 13 and patient had great fear of fall and was unable to ambulate and perform mobility and transfer activities independently.

\section{Research design}

Design used was a single-subject design. Patient was assessed at baseline, after 2 weeks, 6 weeks and 8 weeks of virtual reality balance training.

\section{Procedure}

Patient was selected because he was having obvious balance deficit, fear of fall and depression. Patient performance was assessed in 2 sessions using berg balance scale for dynamic balance, gait dynamic index to assess gait, time up and go test, five times sit to stand test for mobility assessment and fall efficacy scale for fall reduction. Patient was assessed at baseline and then after 2 weeks, 4 weeks, 6 weeks and 8 weeks. Berg balance was used to assess dynamic balance, time up and go for mobility assessment. 14 tasks were performed in berg balance scale. (1) Sit to stand (2) standing unsupported (3) sitting with back unsupported (4) Standing to sitting (5) transfer (6) standing unsupported with eyes close (7) standing unsupported feet together (8) reaching forward (9) picking up object for floor (10) turning back (11) turning 360 degree (12) alternate foot step (13) standing foot front (14) standing on one leg. Each task was scored ranging from 0-4 with 0 being minimum score and 4 maximum score. Time up and go is a simple mobility test, patient is asked to get up from the chair walk 
a distance of 3 meters then turn back and walk back to the chair and sit down. Five time sit to stand is performed by asking the patient to stand up from the sitting position to complete erect position and repeat 5 times. 8 tasks are performed in gait dynamic index to challenge gait. Fall efficacy scale was designed to estimate fear of fall. Low score on FES indicates a reduced risk of fall and high rate indicates greater risk of fall in activities of daily living. (4) It has an excellent test retest reliability of $(\mathrm{ICC}=0.97)$. It consists of 10 different questions related to ADLs.

\section{Apparatus}

Virtual reality balance training was given to the patient using Xbox 360 with kinect. Xbox 360 Kinect is a camera system that is controller free camera system that use infrared technology. Kinect detects wave of the hand and body movements. The software tracks 48 body points. Camera tilts upward and downward and detects body movements and follows user around the range of the camera. ${ }^{5} 20,000$ leaks game was used to train patient. In this game the player's avatar is in a glass cube under water. Fish, crabs, sharks \& other sea creatures cause cracks and holes in the cube. Player uses limbs, hands, feet and head to plug the holes. Each game consists of three waves. The name is a reference to the novel "20,000 Leagues under the Sea" by Jules Verne (Figure 1).

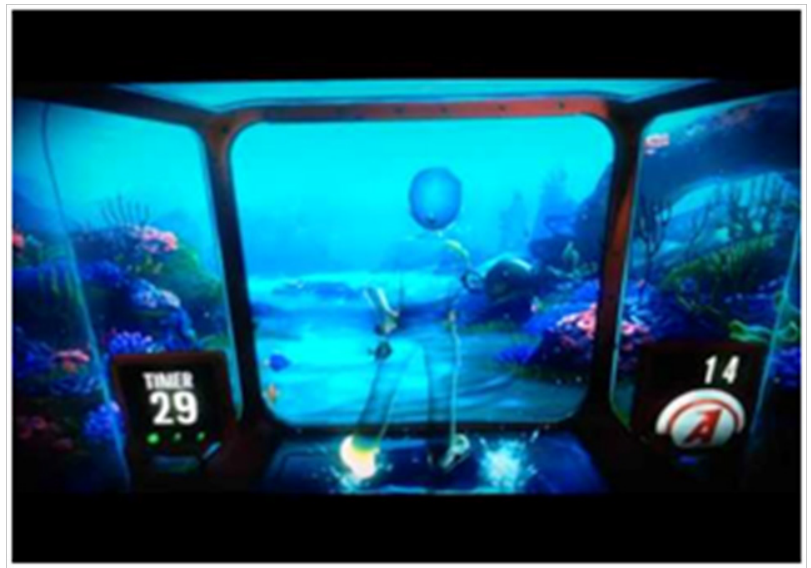

Figure I Xbox 360: 20,000 Leaks.

\section{Training}

The patients initially used his right hand to plug the leaks. While doing so he moved right to left to reach the plugs. At the start it was more challenging and patient faced great efforts to plug the leaks. While playing, he moved out of base of support but managed to balance. With few consecutive sessions he was able to perform more effectively and comfortably and enjoyed his training. Game was played 3 times per session with resting period of 5 minutes in between. Session was given 4 days per week for total 8 weeks.

\section{Results}

Berg balance scale score of the patient improved from 13 to 42 after 8 weeks of training. Figure 2 shows that at baseline when patient started playing Xbox games the score for BBS was 13. After 2 weeks the score improved from 13 to 18 . With another 2 weeks of training the score improved to 21 with prominent improvement in turning back and standing unsupported with eyes closed. After 6 weeks of treatment the score improved to 38 with maximum deficit in standing on one leg. After 8 weeks of VR training the score improved to 42. Two components of berg balance scale were not found to be improving, these were alternate foot stepping and standing on one leg. Time up and go test reading was $1 \mathrm{~min} 35 \mathrm{sec}$ at initial assessment and improved to $45 \mathrm{sec}$ after 8 weeks of training. Reading of 5 times sit to stand test improved from $1 \mathrm{~min} 15 \mathrm{sec}$ to $40 \mathrm{sec}$.

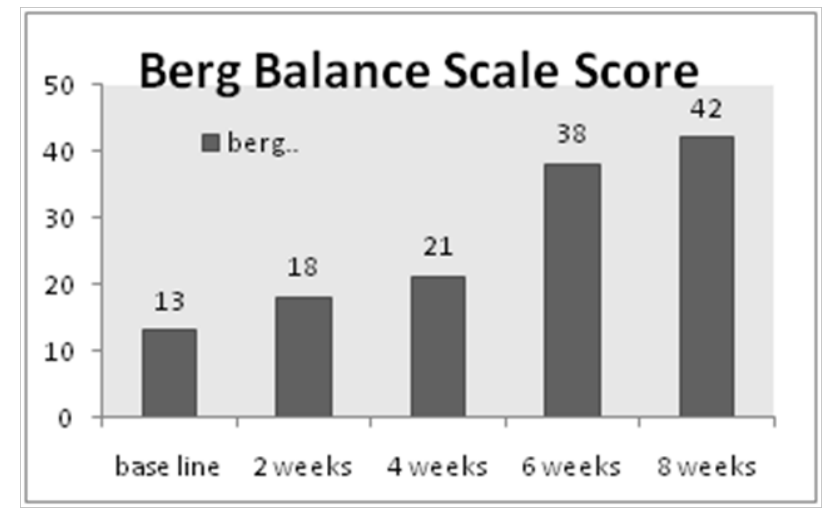

Figure 2 Berg Balance Scale Scores Over 8 Weeks.

(Figure 3) shows gait dynamic index scores improving from 1 to 13 over period of eight weeks of training. Fall efficacy scale scored 82 at baseline and after 8 weeks it was improved to 39 .

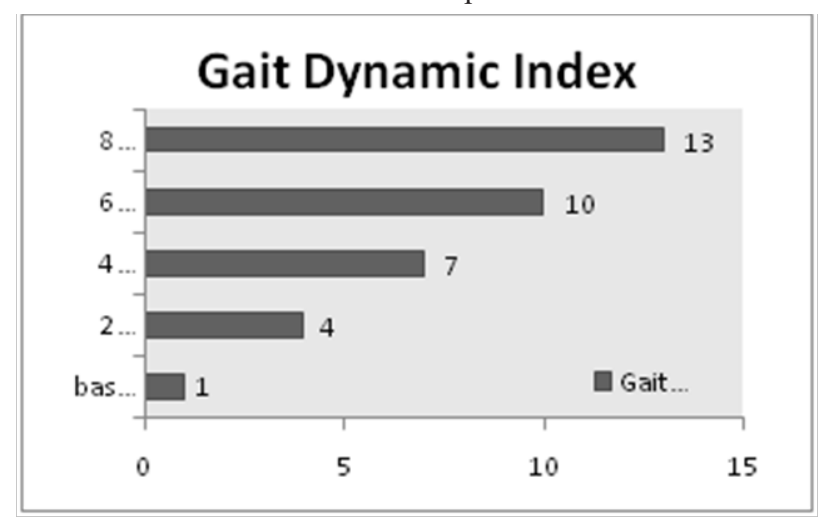

Figure 3 Gait Dynamic Index Scores Over 8 Weeks.

\section{Discussion}

Virtual reality and interactive video gaming extensively used in the treatment of stroke patient As Laver KE et al in their systemic review concluded that virtual reality and interactive video gaming have more effectiveness as compare to the conventional means of treatments it is also examined that virtual reality and interactive video gaming have favorable effect on upper limb function and ADL function when used alongside usual care. ${ }^{6}$

The study reported ambiguous evidence about the effectiveness of virtual reality and interactive video gaming on grip strength, gait speed or global motor function. It is still debatable that which of the characteristics of virtual reality have greater impact, it is also unclear that effects of the characteristics are adequate in long term. de Rooij et al. ${ }^{7}$ also supported the evidence that VR training is more beneficial than conventional balance or gait training. ${ }^{7}$ The study of Silva et al. ${ }^{8}$ revealed that virtual reality parallel with kinesiotherapy and learning transfer exercises had an efficacious impact on stroke individuals. ${ }^{8}$

\section{Conclusion}

The present concludes that Virtual training is capable of improving balance and helps the patient to overcome fear and hesitations regarding mobility and ambulation in the way when patient involves in the virtual reality he moved unintentionally and freely, which eventually helps to improve his balance, mobility and reduce his fear of fall. Virtual reality also improves the confidence of Stroke patients 
who are psychologically discouraged to perform their daily activities independently. Patient indulges in the environment and performs activities unconsciously he previously was afraid of. Further detailed studies are suggested to explore the effectiveness of virtual reality on fear of fall.

\section{Acknowledgments}

None.

\section{Conflicts of interest}

None.

\section{References}

1. Tyson SF, DeSouza LH (2004) Reliability and validity of functional balance tests post stroke. Clinical rehabilitation 18(8): 916-923.

2. Laver K, George S, Thomas S, Deutsch JE, Crotty M (2012) Virtual reality for stroke rehabilitation. Stroke 43(2): e20-e21.
3. Adamovich SV, Fluet GG, Tunik E, Merians AS (2009) Sensorimotor training in virtual reality: a review. Neuro Rehabilitation 25(1): 29-44.

4. Kwakkel G, van Peppen R, Wagenaar RC, Dauphinee SW, Richards C, et al. (2004) Effects of augmented exercise therapy time after stroke a meta-analysis. Stroke 35(11): 2529-2539.

5. DePriest D, Barilovits K (2011) LIVE: Xbox Kinect $\odot$ s virtual realities to learning games. TCC Worldwide Online Conference, Hawaii, USA.

6. Laver KE, George S, Thomas S, Deutsch JE, Crotty M (2015) Virtual reality for stroke rehabilitation. Cochrane Database Syst Rev 9: CD008349.

7. de Rooij IJ, van de Port IG, Meijer J-WG (2016) The effect of virtual reality training on balance and gait ability in patients with stroke: a systematic review and meta-analysis. Phys Ther 96(12): 1905-1918.

8. Silva WHS, Lopes GLB, Yano KM, Tavares NSA, Rego IAO, et al. (2015) Effect of a rehabilitation program using virtual reality for balance and functionality of chronic stroke patients. Motriz: Revista de Educação Física 21(3): 237-243. 